\title{
Bilateral Retinal Vasculitis in Association with Systemic Sclerosis sine Scleroderma. A case report and brief review of the literature.
}

Francisco Manuel Hermoso Fernandez ( $\sim$ framaherfer@gmail.com )

Hospital Universitario San Cecilio https://orcid.org/0000-0003-1710-989X

Norberto Ortego-Centeno

Hospital Universitario San Cecilio

Jose Luis Callejas-Rubio

Hospital Universitario San Cecilio

Jose L. García-Serrano

Hospital Universitario San Cecilio

\section{Short Report}

Keywords: systemic, sclerosis, scleroderma, sine, rare, report

Posted Date: December 2nd, 2021

DOI: https://doi.org/10.21203/rs.3.rs-1104339/v1

License: (c) (i) This work is licensed under a Creative Commons Attribution 4.0 International License. Read Full License 


\section{Abstract}

Background: Systemic sclerosis sine scleroderma is a very rare subset characterized by the total or partial absence of cutaneous manifestations of systemic sclerosis with the occurrence of internal organ involvement and serologic abnormalities. The purpose of this article is to report a case showing a very rare association of bilateral retinal vasculitis (RV) and systemic sclerosis sine scleroderma (ssSSc). We show the description of a case report and revision of other medical histories, prospective and retrospective studies.

Findings: A 40-year-old woman presented at our Hospital for blurry vision with her left eye (OS). Her bestcorrected visual acuity was $20 / 20$ in both eyes. Funduscopy examination showed bilateral occlusive vasculitis with proliferative retinopathy and vitreous hemorrhages. Retinography showed temporary sheathed and some occluded veins, surrounded by large number of retinal hemorrhages in both eyes. In fluorescein angiography were found areas of not perfused retina, also with telangiectasia and staining of retinal vessel's walls. We found isolated microaneurysms in these ischemic areas. The ophthalmological treatment was confluent photocoagulation of the ischemic retina of both eyes, and vitrectomy of the right eye to treat his vitreous hemorrhage. The systemic treatment was corticosteroids and Adalimumab during two years; afterwards the stability was achieved. There was a lack of response to cyclosporine and azathioprine. The diagnosis comes years later after presenting: anticentromere antibodies, scleroderma pattern on capillaroscopy and esophageal manometry showing esophageal dysmotility with absent contractility.

Conclusion: We report a rare association of RV and sclerosis sine scleroderma. RV can precede the development of ssSSc by several years.

\section{Introduction}

Systemic sclerosis (SSc) is a complex connective tissue disease of unknown etiology with multiorgan involvement and heterogeneous clinical manifestations ${ }^{1}$. The disease has 2 main subsets, diffuse and limited forms. The subset known as systemic sclerosis sine scleroderma (ssSSc) is a very rare subset characterized by the total or partial absence of cutaneous manifestations of systemic sclerosis with the occurrence of internal organ involvement and serologic abnormalities ${ }^{2}$. Diffuse cutaneous systemic sclerosis (dcSSc) is associated with anti-topoisomerase (ATA) whereas limited cutaneous (IcSSc) and sine scleroderma (ssSSc) are mainly associated with anti-centromere antibody (ACA) ${ }^{3}$.

Most commonly reported ocular pathologies include eyelid stiffness, that is seen in more than $50 \%$ of the cases and results from deposition of type I collagen in the dermis. Keratoconjunctivitis sicca is the second most common problem seen in $50 \%$ of the affected patients. Cases of conjunctivitis, episcleritis, anterior uveitis, and hypertensive retinopathy have also been reported ${ }^{4}$. This report describes the clinicopathologic findings in a young woman who presented a bilateral RV accompanied by a sclerosis sine scleroderma. 


\section{Case Presentation}

A 40-year-old woman presented at our Hospital for blurry vision since this morning. On ophthalmic examination, her best-corrected visual acuity (Snellen chart) was ${ }^{20} / 20$ in both eyes. On slit-lamp examinations nothing unusual was observed. Fundus evaluation showed an inferior temporal branch retinal vein occlusion, retinal neovascularization, venous tortuosity and vitreous haemorrhage in her left eye and superior temporal vasculitis in his right eye. Retinography showed temporary sheathed and some occluded veins, surrounded by a large number of retinal hemorrhages in both eyes (figure 1). In fluorescein angiography were found extensive areas of not perfused retina, with telangiectasia and staining of retinal vessels walls. We found isolated microaneurysms in these ischemic areas and absent in the rest of the retina (Figure 2).

The study of infectious causes of uveitis was negative (tuberculosis, syphilis, Lyme disease). The general study pointed to a vasculitis of immunological origin: Raynaud phenomenon, pathological capillaroscopy, Positive ANAS 1/640, and underwent retinal laser photocoagulation over the ischaemic retina in both eyes. There was a lack of response to cyclosporine and azathioprine with persistent vascular sheaths and bleeding repeat vitreous. Right eye vitrectomy to treat his persistent vitreous hemorrhage. The systemic treatment was corticosteroids and Adalimumab for two years, and the stability of the condition was achieved.

The diagnosis comes after referring gastroesophageal reflux and heartburn, an esophageal manometry was performed, which showed esophageal dysmotility with absence of movement and a positive result for anti-centromere antibodies. The intestinal vasculitis remitted with corticosteroid boluses. The diagnosis of systemic sclerosis sine sclerodermia was reached.

At this time we also noticed the telangiectasias in the vessels of the retina, which we had found when performing fluorescein angiography. Six months later, the general treatment was discontinued due to the inactivity of the vasculitis, disappearance of vascular sheaths, and retinal hemorrhages.

The patient has been followed up every month over a period of 2 years and the clinical features of the treated lesions were unchanged during this period of follow-up.

\section{Discussion}

To our knowledge, this is the first reported case of a combination of systemic sclerosis sine sclerodermia and bilateral retinal vasculitis.

Systemic sclerosis is a rare connective tissue disorder characterized by abnormal fibroblast proliferation leading to deposition of extracellular matrix in the skin, blood vessels or viscera. This results in the stiffness of the connective tissue structures in the skin or body organs. Like other autoimmune diseases, it is characterized by the interaction of inflammatory cytokines and antibodies ${ }^{4}$. It is generally accepted that SS is characterized by prominent vascular involvement that is not limited to the peripheral 
microcirculation of the skin, but is also observed in the heart, lungs, kidneys, gastro-intestinal tract and eyes ${ }^{5}$. Depending on the presentation of this disease we can separate three different types, diffuse cutaneous systemic sclerosis (dcSSc), which is associated with anti-topoisomerase (ATA) whereas limited cutaneous (IcSSc) and sine scleroderma (ssSSc) are mainly associated with anti-centromere antibody $(\mathrm{ACA})^{3}$.

The sclerosis systemic varies from the mildest syndrome or CREST syndrome to the most severe or diffuse syndrome. The CREST syndrome is called for calcinosis, Raynaud's phenomenon, esophageal dysmolity, sclerodactyly, and telangiectasia. In these patients, visceral involvement is less common than with diffuse sclerosis systemic. This syndrome appears to be relatively benign and more slowly progressive. Retinal vasculitis associated with CREST syndrome ${ }^{6}$ with poor response to immunosuppression has been described. In our case, despite immunosuppressive treatment, retinal vasculitis was recurrent with new hemorrhages and sheathing in areas already treated; on the other hand, with the effect of corticosteroid bolus decreased.

Histological studies in patients with SS have demonstrated that choroidal vessels are grossly affected and present endothelial cell damage, basement membrane thickening and absence of pericytes ${ }^{7}$. Persistence of such widespread endothelial dysfunction in systemic vasculitis appears to have long-term consequences, leading to the acceleration of premature ischaemic disease ${ }^{8}$.

Systemic sclerosis has been reported to be associated with several ocular abnormalities such as conjunctivitis, episcleritis, anterior uveitis, and hypertensive retinopathy. However, there is no previous report of systemic sclerosis sine scleroderma associated with bilateral retinal vasculitis. In conclusion, it is important to think that a retinal vasculitis can be caused by a rare condition such as systemic sclerosis sine scleroderma.

Retinal vasculitis is an inflammation of retinal blood vessels that progress from vessel closure to ischemia and subsequent retinal neovascularization ${ }^{9-10}$. It is characterized by intermittent sheathing of the involved eyes and aneurysms, showing vascular staining in fluorescein angiography. As a clinical entity, a course with recurrent retinal and vitreous hemorrhages, such as Eales's disease treated with retinal laser and vitrectomy. Based on that, it could be of great significance to look for markers, such as anti-centromere antibodies, and to perform capillaroscopy. Retinal vasculitis can precede the development of ssSSc and internal organ involvement by several years, including cardiac involvement which represents the most important clinical feature with a high morbidity and mortality rate ${ }^{11}$.

\section{Conclusion}

Systemic sclerosis sine scleroderma can occur with retinal vasculitis that may be underdiagnosed. Retinal vasculitis can precede the development of systemic sclerosis disease by years and raynaud phenomenon and a pathological capillaroscopy with anticetromere antibodies can alert us to look for possible systemic and organic involvement of the disease. 


\section{Abbreviations}

RV: Retinal vasculitis

ssSSc: Systemic sclerosis sine scleroderma

SSc: Systemic sclerosis

ATA: Anti-topoisomerase

IcSSc: Systemic sclerosis limited cutaneous

dcSSc: Diffuse cutaneous systemic sclerosis

DMII: Diabetes mellitus type 2

\section{Declarations}

\section{Conflict of Interest statement}

None of the authors has any financial/ conflict of interest to disclose.

\section{Informed Consent and Human and Animal Rights statement}

Informed consent has been obtained from all individuals included in this study.

The patient offered her consent for the publication of her identifiable details in relation to the article "Bilateral Retinal Vasculitis in Association with Systemic Sclerosis sine Scleroderma. A case report and brief review of the literature" in Journal of Ophthalmic Inflammation and Infection

He offered her consent for the publication of her identifiable details, including photograph(s) and/ or videos and/ or case history and/ or details in the text to be published in the above-mentioned journal. She discussed this consent form with Francisco Manuel Hermoso Fernández, the author of the paper.

\section{Authors' contributions}

All author here cited contributed to conception and design of the study, acquisition of data, manuscript drafting and approval of the final version.

\section{Authorization for the use of human subjects}

Ethical approval: The research related to human use complies with all the relevant national regulations, institutional policies, is in accordance with the tenets of the Helsinki Declaration, and has been approved by the review board of San Cecilio University Hospital, Granada, Spain.

\section{Acknowledgements}


None.

\section{Sources of Funding}

Authors have not received founding from any organization related (National Institutes of Health $(\mathrm{NIH})$; Welcome Trust; Howard Hughes Medical Institute (HHMI).

\section{Disclosures}

The authors declare that they have no interest in relation to this article.

\section{References}

1. Pattanaik D, Brown M, Postlethwaite BC, Postlethwaite AE (2015) Pathogenesis of systemic sclerosis. Front Immunol 6:272

2. Kucharz EJ, Kopeć-Mędrek M (2017) Systemic sclerosis sine scleroderma. Adv Clin Exp Med 26(5):875-880

3. Espinosa G, Del Guillén A, Freire M et al (2020) Serodiscordant patients with systemic sclerosis: when antibody does not correspond to skin involvement.Clinical and Experimental Rheumatology.

4. Choudhary MM, Hajj-Ali RA, Lowder CY (2014) Gender and ocular manifestations of connective tissue diseases and systemic vasculitides. Journal of ophthalmology. ;2014

5. Ingegnoli F, Gualtierotti R, Pierro L, Del Turco C et al (2015) Choroidal impairment and macular thinning in patients with systemic sclerosis: the acute study. Microvasc Res 97:31-36

6. Yang S, Kopplin LJ, Rosenbaum JT (2018) Retinal vasculitis associated with CREST syndrome. American journal of ophthalmology case reports 10:185-188

7. Farkas TG, Sylvester V, Archer D (1972) The choroidopathy of progressive systemic sclerosis (scleroderma). Am J Ophthalmol 74(5):875-886

8. Buckley CD, Rainger GE, Nash GB, Raza K (2005) Endothelial cells, fibroblasts and vasculitis. Rheumatology 44(7):860-863

9. Gascon P, Jarrot P-A, Matonti F, Kaplanski G (2018) Vascularites rétiniennes et maladies systémiques. La Revue de Médecine Interne 39(9):721-727

10. Rosenbaum JT, Sibley CH, Lin P (2016) Retinal vasculitis. Curr Opin Rheumatol 28(3):228

11. Zulian F, Lanzoni G, Castaldi B, Meneghel A, Tirelli F, Zanatta E et al (2021) Systemic sclerosis sine scleroderma in children.Rheumatology [Internet].Oct 4

\section{Figures}




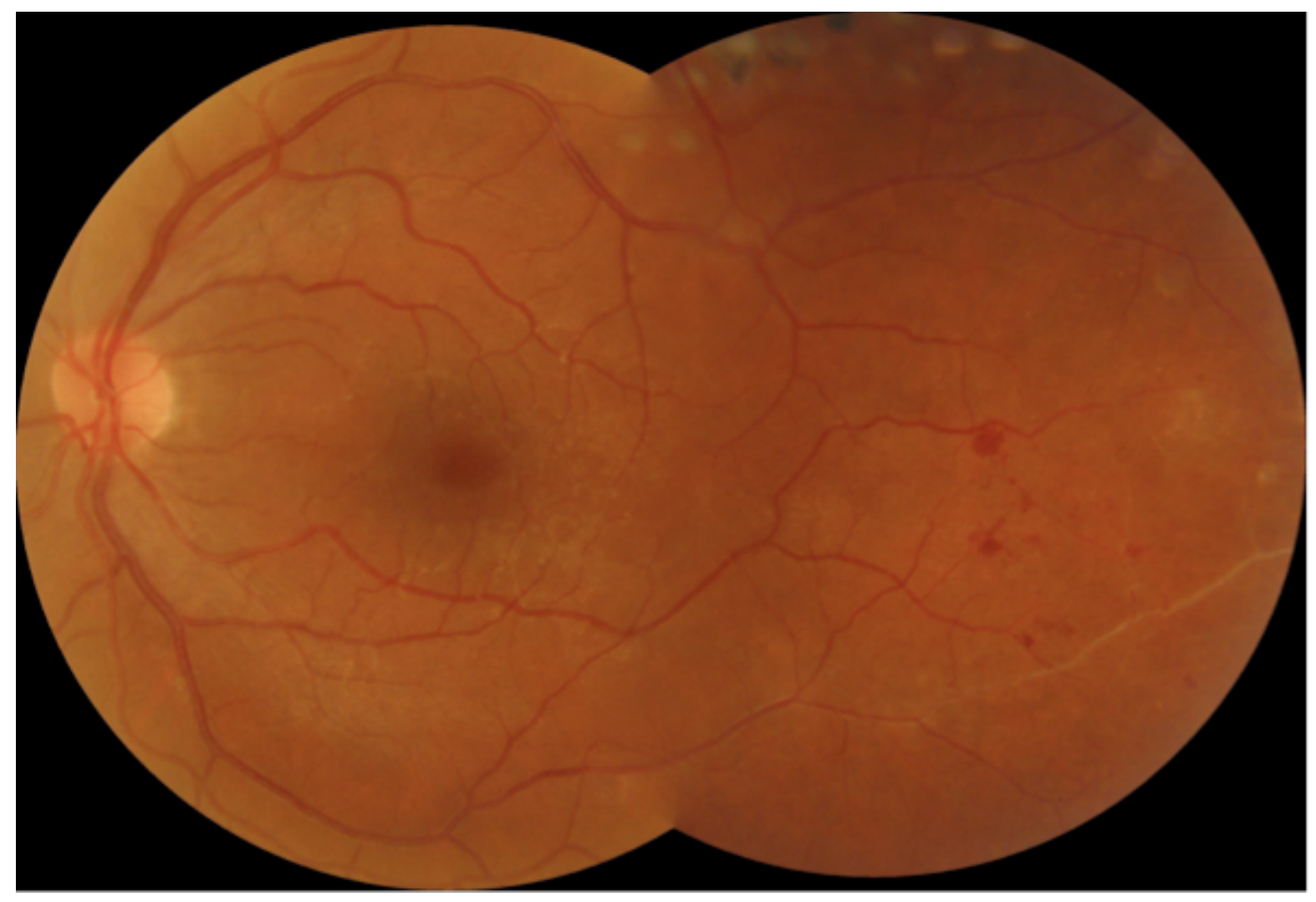

\section{Figure 1}

In left eye: We see sheathed veins, which are continued with vascular occlusions, telangiectasia and hemorrhages. 


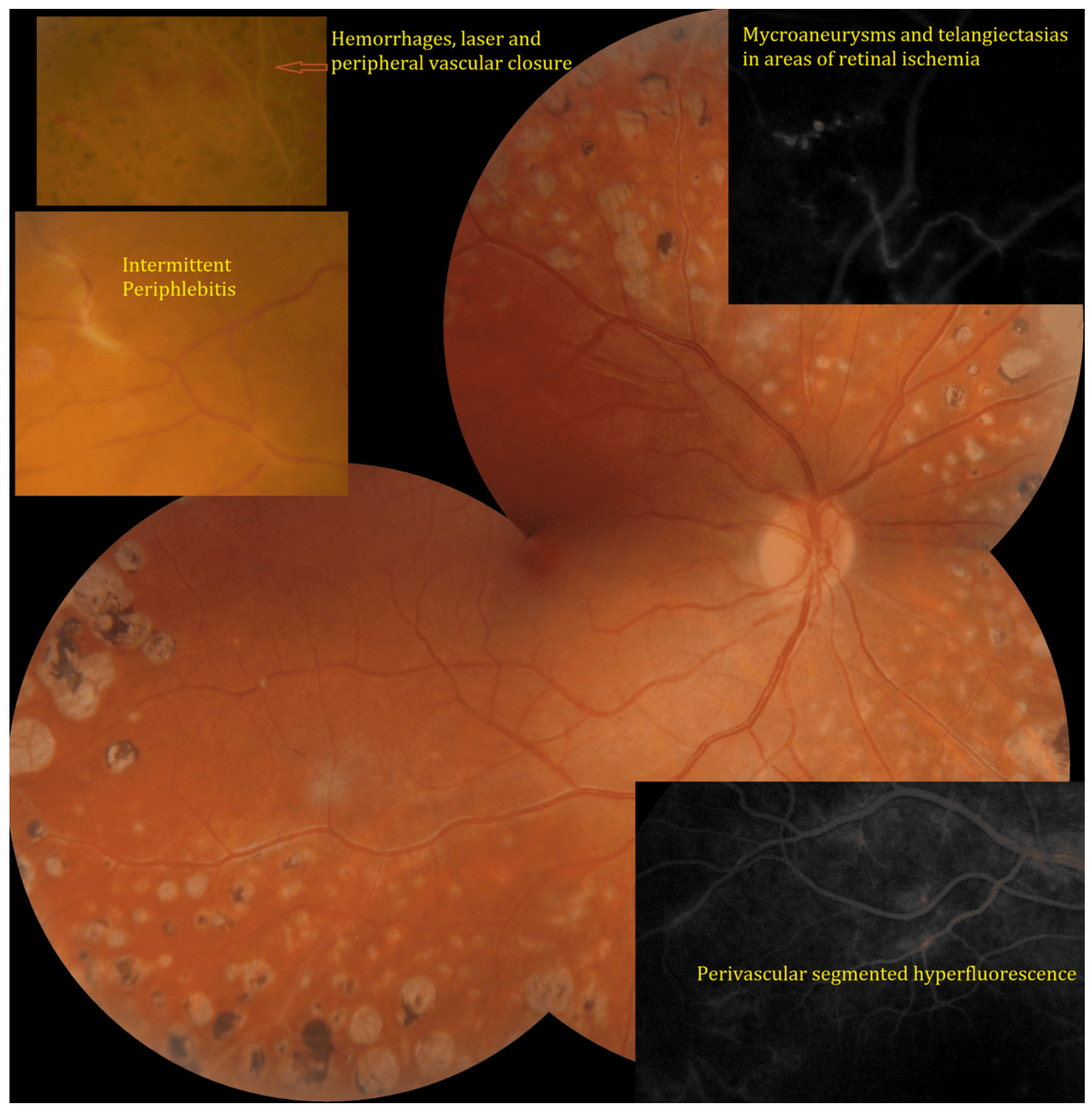

Figure 2

Panretinal photocoagulated right eye fundus with areas of chronic venous sclerosis (white linear opacity adjacent to the vein). Photomontage of main lesions found in the periphlebitis: Segmented periphlebitis with perivascular hyperfluorescence, hemorrhages, and vascular and capillary closures. They originate areas of retinal ischemia with microaneurysms and focal telangiectasia, the origin of neovessels and vitreous hemorrhages that recur. 